\title{
Detection of spring wheat plants affected by powdery mildew using hyperspectral survey data
}

\author{
Tamara A. Gurova ${ }^{1}$, Ol'ga A. Dubrovskaja ${ }^{2}$, Oleg V. Elkin ${ }^{1}$, Lev V. Maximov ${ }^{3}$, \\ Igor A. Pestunov ${ }^{2}$ and Viktor G. Altukhov ${ }^{1}$ \\ ${ }^{1}$ Siberian Federal Scientific Center of Agrobiotechnology RAS, Krasnoobsk, Russia \\ ${ }^{2}$ Federal Research Center for Information and Computational Technologies SB RAS, Novosibirsk, Russia \\ ${ }^{3}$ Institute of Automation and Electrometry SB RAS, Novosibirsk, Russia
}

\begin{abstract}
In laboratory experiments, spectral characteristics of three varieties of Siberian selection spring wheat affected under field conditions by powdery mildew (Blumeria graminis (DC.) Speer) were obtained using hyperspectral camera. The variety specificity of the reflectivity of wheat leaves affected by powdery mildew with the same severity has been established. A change in the leaves reflectivity depending on the severity was revealed. The most informative spectral indicator (index) for the powdery mildew detection has been determined.
\end{abstract}

\section{Keywords}

Wheat diseases, powdery mildew, hyperspectral data, spectral characteristics, vegetational indices.

\section{Introduction}

Spring soft wheat is traditionally one of the main food grain crops in the world. Its crop share in the total volume of grain production in Russia is $62.2 \%$. One of economically significant wheat pathogens is powdery mildew (Blumeria graminis (DC.) Speer).

Wheat powdery mildew is highly destructive disease widespread worldwide, in Russia, and in Western Siberia [1, 2]. Disease destructiveness comes out in decreasing of assimilative leaf surface, which leads to their premature drying, ear formation setback, stem sclerenchyma attenuation, ear length and grain content reduction, grain shriveling and ear destruction. In some years, shortfall in wheat production can be more than $15-20 \%$, and in the epiphytotic years beyond the $50 \%[3,4]$.

Powdery mildew pathogen Blumeria graminis progresses mainly on actively vegetative young plants. Symptoms of disease appear on leafs, stem internodes and gluma as white, loose powdery coating, consisting of the fungus conidiophores and conidia [5].

The existing standard methods of phytosanitation monitoring are based on visual diagnosis of wheat diseases development and propagation. Crops inspection for powdery mildew infestation coincides with periods of sprouts - tillering and booting - ear formation. Examination of the plants conducts at 10-15 points along diagonal of the inspected field. The number of viewed plants is counted [6]. Degree of disease development is determined as average measure of plants

SDM-2021: All-Russian conference, August 24-27, 2021, Novosibirsk, Russia $\bigoplus$ guro-tamara@yandex.ru (T. A. Gurova); olga@ict.nsc.ru (O. A. Dubrovskaja)

(c) (i) $\odot 2021$ Copyright for this paper by its authors. Use permitted under Creative Commons License Attribution 4.0 International (CC BY 4.0).

CEUR Workshop Proceedings (CEUR-WS.org) 
infestation, which takes into account the number (percentage) of diseased plants and severity (score). Depending on the crop and acreage size, which is often very large, this method can be quite laborious.

Currently, active investigations are carried out to develop methods and approaches for plant diseases remote diagnosis based on using hyperspectral visualization of plants reflectivity $[7,8]$. It was demonstrated that hyperspectral visualization methods can be used in detection and diagnosis of powdery mildew $[9,10]$. Various spectral indicators of plants diseased leafs have been established. According to hyperspectral data obtained for one variety of spring wheat, the index (PMI) was determined, which identifies powdery mildew [11, 12].

The purpose of our research is to reveal the informational content of spectral characteristics of reflectivity of Siberian varieties spring wheat leafs for powdery mildew infestation detection (recognition), taking into account the varieties genotypes and plants severity.

\section{Materials and methods}

Investigations were carried out in laboratory conditions. Wheat plants grown in a natural infection background. Samples of healthy wheat plants and wheat plants diseased by powdery mildew (the pathogen Blumeria graminis (DC) Speer) were collected at the test fields of Siberian Research Institute of Plant Cultivation and Breeding (Branch of Institute of Cytology and Genetics of Siberian Branch of Russian Academy of Sciences) and Siberian physico-technical institute (Siberian federal scientific Center of Agrobiotechnology of Russian Academy of Science). Location: Novosibirsk region, Novosibirsk district, Michurinskij and Krasnoobsk workers settlements. Wheat plants samples of varieties Sibirskaya 12, Sibirskaya 21, Novosibirskaya 44 were collected at the periods of tillering and booting - ear formation.

The following variants were evaluated:

1) control (green leaf without visible disease markers);

2) powdery mildew with visible disease markers: light and medium severity $10-50 \%$, and severe severity $50-100 \%$.

Representational selection is $20-25$ plants in each experiment version. Hyperspectral images of wheat for analysis were captured by hyperspectral camcorder Photonfocus MV1-D2048x1088HS05-96-G2-10 in the 475-900 nm waveband. Spectral resolution of the camcorder is $3 \mathrm{~nm}$ and includes 150 bands. Spatial resolution of the sensor is $2088 \times 1088$ pixels.

Visual quality precheck of hyperspectral images was performed using Photonfocus Studio software. For bulk image processing Python library images were used. Data extraction by spectral lines intensity and their statistical analysis were conducted by ENVI software. Spectra were smoothed using Savitsky - Golay filter. When spectral curves obtaining, the images were segmented and selected segments spectral brightness average values were utilized by several images.

For purposes of plants analysis for the powdery mildew detection, 11 vegetation indices were calculated: NBNDVI, RVSI, PSRI, NRI, PRI, SIPI, PhRI, TCARI, MTVI1, TVI, PMI, which were described earlier [13]. 


\section{Results and discussion}

The specificity of curves of vegetation spectral reflectance, which allows recognizing crop diseases with sufficient accuracy, depends on the pathogenesis type as a reaction of plants to the introduction and development of disease pathogen. Vegetational objects are characterized by low reflectivity in the blue and red spectrum regions, moderate increase in green region, and strongly pronounced maximum in near infrared band.

Figure 1 shows the obtained spectral curves of plants leaves of Sibirskaya 12 and Novosibirskaya 44 wheat varieties, healthy and infected by powdery mildew (Blumeria graminis $(D C)$ Speer) with the same severity.

For all tested varieties in case of normalized spectra, curve shapes of healthy and diseased leaves are similar. Reflectivity ratio value of healty leaf in extreme red region of visible spectrum $(470-680 \mathrm{~nm})$ was at the minimum, which is due to light absorption by pigments (chlorophyll). In close infrared region leaves reflectivity was rising, which is associated with internal light scattering by mesophyll. Sharp increase of the spectrum characteristics values was observed on the red boundary and near infrared portions of the spectrum $(690-740 \mathrm{~nm})$. Starting from the peak values in the $760 \mathrm{~nm}$ region, with wavelengths increasing in the range of $760-880 \mathrm{~nm}$, a slight reflectivity decreasing was registered in all cases. It may be caused by plants moisture content.

Leaves of all tested wheat varieties diseased by powdery mildew differed from healthy ones by higher reflectivity values in the visible spectrum $(480-750 \mathrm{~nm})$ and lower in near infrared spectrum range $(750-880 \mathrm{~nm})$. It indicated their physiological state deterioration. We have established the variety specificity of the reflectivity of wheat leaves affected by powdery mildew with the same severity. Thus, the relative changes of healthy and diseased leaves reflectivity of sensitive variety Sibirskaya 12 are more pronounced in comparison with resistant variety Novosibirskaya 44 both in the visible and near infrared spectrum ranges. Herewith the relative changes of reflectivity ratios were 138.0, 129.0 and $11.8 \%$ for Sibirskaya 12 variety and 14.6, 76.8 and $4.0 \%$ for Novosibirskaya 44 variety at the points $478 \mathrm{~nm}, 680 \mathrm{~nm}$ and $765 \mathrm{~nm}$ respectively. Protection systems of Novosibirskaya 44 variety are more lable, which reduces distructive process development in this variety during powdery mildew pathogenesis.
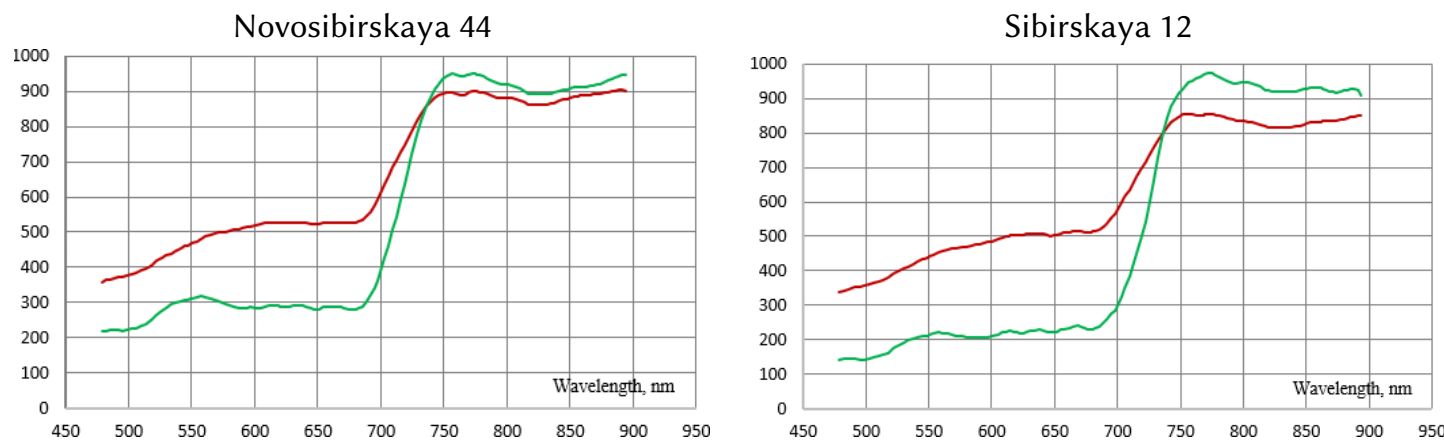

Figure 1: Spectral curves of healthy leaves (green curve) and leaves infected by powdery mildew (Blumeria graminis (DC) Speer) of two wheat varieties with $50-100 \%$ severity (red curve). 
As a result of analysis of spectral curves of wheat leaves diseased by powdery mildew with different severity, leaves reflectivity changing was established depending on disease severity (Figure 2). The stronger severity (50-100\%) the higher leaves reflectivity in visible spectrum region. The opposite result was observed in near infrared region: the higher leaves reflectivity, the lower severity $(10-50 \%)$. Areas with the greatest differences between healthy

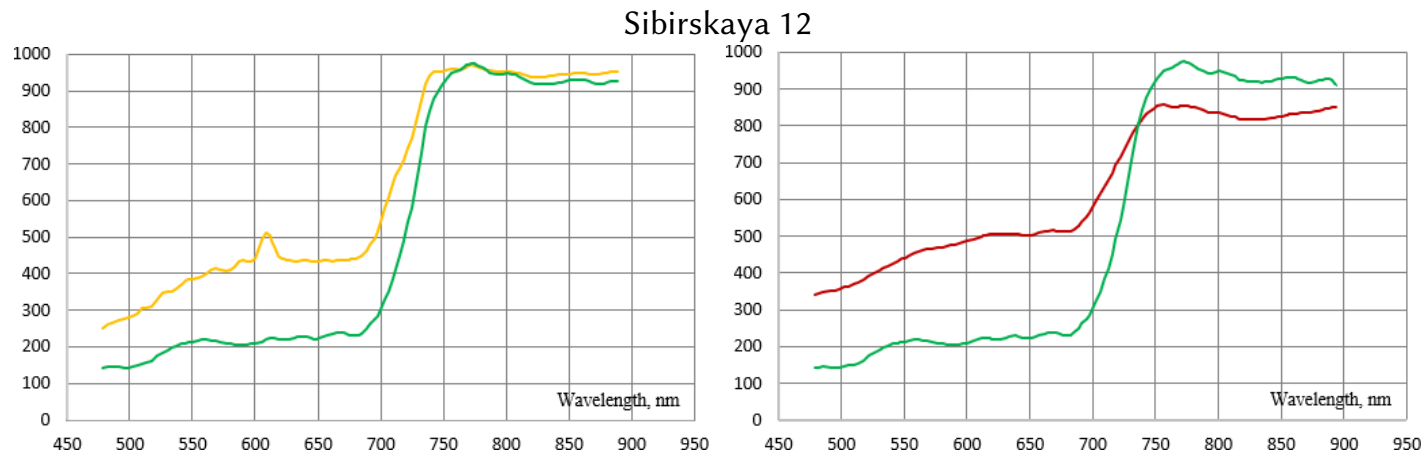

Figure 2: Spectral curves of healthy leaves (green curve) and leaves infected by powdery mildew (Blumeria graminis (DC) Speer) with 10-50\% (yellow curve) and 50-100\% (red curve) severity.

\section{Table 1}

Vegetational indices for powdery mildew identification on wheat leaves.

\begin{tabular}{|c|c|c|}
\hline Index & Abbreviation expansion & Formulary \\
\hline & & $R_{570}-R_{670}$ \\
\hline NRI & Nitrogen Reflectance Index & $\overline{R_{570}+R_{670}}$ \\
\hline NBNDVI & Narrow-Band Normalized & $R_{850}-R_{680}$ \\
\hline INDINDVI & Difference Vegetation Index & $\overline{R_{850}+R_{680}}$ \\
\hline RVSI & $\begin{array}{l}\text { Red-Edge Vegetation } \\
\text { Stress Index }\end{array}$ & $\frac{R_{712}+R_{752}}{2}-R_{732}$ \\
\hline PSRI & $\begin{array}{l}\text { Plant Senescence } \\
\text { Reflectance Index }\end{array}$ & $\frac{R_{\text {Red }}-R_{\text {Green }}}{R_{\text {NIR }}}=\frac{R_{678}-R_{500}}{R_{750}}$ \\
\hline PMI & Powdery Mildew Index & $\frac{R_{515}-R_{698}}{R_{515}+R_{698}}-0.5 R_{738}$ \\
\hline PRI & $\begin{array}{l}\text { Photochemical/Physiological } \\
\text { Reflectance Index }\end{array}$ & $\frac{R_{531}-R_{570}}{R_{531}+R_{570}}$ \\
\hline SIPI & $\begin{array}{l}\text { Structural Independent } \\
\text { Pigment Index }\end{array}$ & $\frac{R_{800}-R_{445}}{R_{800}+R_{680}}$ \\
\hline PhRI & $\begin{array}{l}\text { Physiological } \\
\text { Reflectance Index }\end{array}$ & $\frac{R_{550}-R_{531}}{R_{550}+R_{531}}$ \\
\hline MTVI1 & $\begin{array}{l}\text { Modified Triangular } \\
\text { Vegetation Index }\end{array}$ & $1.2\left[1.2\left(R_{800}-R_{550}\right)-2.5\left(R_{670}-R_{550}\right)\right]$ \\
\hline TCARI & $\begin{array}{l}\text { Transformed Chlorophyll } \\
\text { Absorption and Reflectance } \\
\text { Index }\end{array}$ & $3\left[\left(R_{700}-R_{670}\right)-0.2\left(R_{700}-R_{550}\right) \frac{R_{700}}{R_{670}}\right]$ \\
\hline TVI & Triangular Vegetation Index & $\begin{array}{l}0.5\left[120\left(R_{\text {Nir }}-R_{\text {Green }}\right)-200\left(R_{\text {Red }}-R_{\text {Green }}\right)\right]= \\
\quad=0.5\left[120\left(R_{750}-R_{550}\right)-200\left(R_{670}-R_{550}\right)\right]\end{array}$ \\
\hline
\end{tabular}




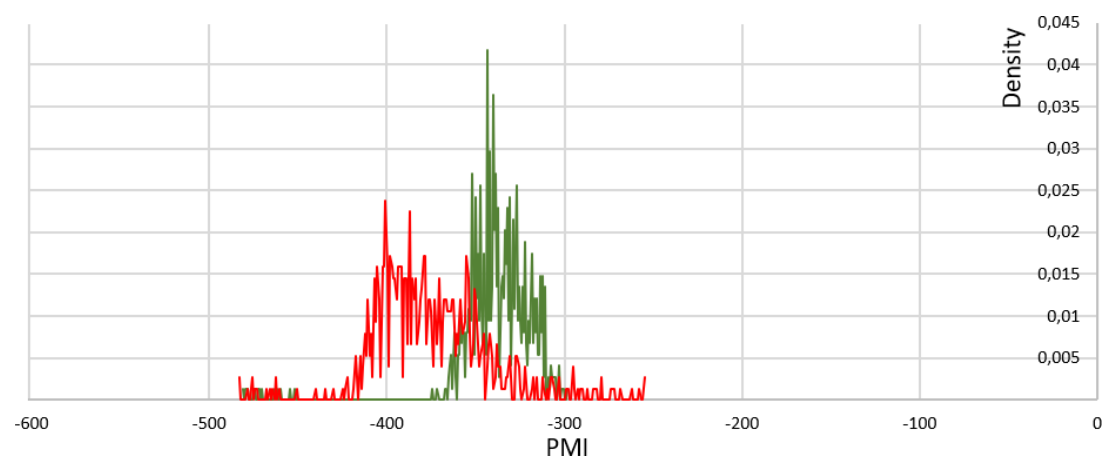

Figure 3: Histogram of PMI (powdery mildew index) vegetation index values: healthy wheat leaves (green curve) and diseased by powdery mildew (Blumeria graminis (DC) Speer) (red curve).

leaves and leaves diseased by powdery mildew with different severity were identified. They are 550-680 $\mathrm{nm}$ and $760-880 \mathrm{~nm}$. The similar results were obtained in analyzing of spectrum characteristics of healthy wheat and wheat diseased by leaf and stem rust, yellow rust, septoria, helminthosporiosis [14].

Since the visible and infrared regions of the electromagnetic spectrum are allied to physiological stress level in plants, it becomes possible to use certain wavebands for plants diseases detection even before visible symptoms appearance.

As follows from the analysis of various vegetational indices previously used in the diagnosis and monitoring of other wheat diseases development [13], along with the basis of spectrum characteristics analysis obtained in laboratory experiment, 11 vegetational indices were selected to identify powdery mildew (Blumeria graminis (DC) Speer). They are presented in Table 1.

Histograms analysis of vegetational indices values shown (Figure 3) that PMI index is most informative for powdery mildew detection on spring wheat leaves by hyperspectral survey data. Equivalent results were found in [12] for powdery mildew identification on spring wheat. To determine informative indicators, we used $10(513 \mathrm{~nm}), 67(685 \mathrm{~nm})$ and $98(781 \mathrm{~nm})$ channels of Photonfocus hyperspectral camcorder.

\section{Conclusion}

As a result of laboratory experiments, processing and analysis of hyperspectral data spectral characteristics of Siberian selection wheat varieties (Novosibirskaya 44, Sibirskaya 21, Sibirskaya 12) of healthy germs and diseased by powdery mildew (Blumeria graminis (DC) Speer) germs were obtained. The variety specificity of the reflectivity of wheat leaves affected by powdery mildew with the same severity has been established. The more resistant the variety, the less the relative changes in leaves reflectivity. A change in the leaves reflectivity depending on the severity was revealed. The stronger severity, the higher leaves reflectivity in visible spectrum region. The less severity, the higher leaves reflectivity in near infrared region. Histograms analysis of 11 vegetational indices which are widely used for different wheat diseases identification showed that PMI index is the most informative indicator in case of powdery mildew detection on leaves using hyperspectral survey data. 


\section{References}

[1] Ray M., Ray A., Dash S., Mishra A., Achary K.G., Nayak S., Singh S. Fungal disease detection in plants: Traditional assays, novel diagnostic techniques and biosensors // Biosens Bioelectron. 2017. Vol. 87. P. 708-723.

[2] Kojshybaev M., Mumindzhanov H. Methodological guidelines for monitoring diseases, pests and weeds in grain crops. Ankara: Food and Agriculture Organization of the United Nations, 2016. 28 p.

[3] Dolzhenko V.I., Vlasenko N.G., Vlasenko A.N. et. al. Zonal systems of protection of spring wheat from weeds, diseases and pests in Western Siberia. Novosibirsk: SSI SibRIAaC, 2014. $124 \mathrm{p}$.

[4] Neklesa N.P. Powdery mildew of cereals // Plant Protection and Quarantine. 2002. No. 5. P. 46-47.

[5] Avetisjan G.A., Avetisjan T.V. Plant protection. Influence of the trace element manganese on the resistance of soft wheat plants to the causative agent of powdery mildew // Bulletin of the SNBG. 2021. Vol. 138. P. 134-138.

[6] Chenkin A.F., Zaharenko V.A., Belozerova G.S. Phytosanitary diagnosis. Moscow: Ear, 1994. $320 \mathrm{p}$.

[7] Forster A., Behley J., Behmann J., Roscher R. Hyperspectral plant disease forecasting using generative adversarial networks // International Geoscience and Remote Sensing Symposium (IGARSS). July 2019. Art. number 8898749. P. 1793-1796. DOI:10.1109/IGARSS.2019.8898749.

[8] Mahlein A.-K., Kuska M.T., Behmann J., Polder G., Walter A. Hyperspectral sensors and imaging technologies in phytopathology: State of the art // Annual Review of Phytopathology. 2018. Vol. 56. P. 535-558. DOI:10.1146/annurev-phyto-080417-050100.

[9] Abdulridha J., Ampatzidis Y., Roberts P., Kakarla S.C. Detecting powdery mildew disease in squash at different stages using UAV-based hyperspectral imaging and artificial intelligence // Biosystems Engineering. 2020. Vol. 197. P. 135-148. DOI:10.1016/j.biosystemseng.2020.07.001.

[10] Zhao J., Fang Y., Chu G., Yan H., Hu L., Huang L. Identification of leaf-scale wheat powdery mildew (Blumeria Graminis F. sp. tritici) combining hyperspectral imaging and an SVM classifier // Plants. 2020. Vol. 9. Is. 8. P. 1-13. DOI:10.3390/plants9080936.

[11] Yao Z.-F., Lei Y., He D.-J. Identification of powdery mildew and stripe rust in wheat using hyperspectral imaging // Spectroscopy and Spectral Analysis. 2019. Vol. 39. Is. 3. P. 969-976. DOI:10.3964/j.issn.1000-0593(2019)03-0969-08.

[12] Lin F., Wang D., Zhang D., Yang X., Yin X., Wang D. Evaluation of spectral disease index PMI to detect early wheat powdery mildew using hyperspectral imagery data // International Journal of Agriculture and Biology. 2018. Vol. 20. P. 1970-1978.

[13] Dubrovskaya O.A., Gurova T.A., Pestunov I.A., Kotov K.Yu. Methods of detection of diseases on wheat crops according to remote sensing // Siberian Herald of Agricultural Science. 2018. Vol. 48. P. 76-89.

[14] Bekmukhamedov N.E., Karabkina N.N. Spectral characteristics change of spring wheat plants infected by fungal diseases // Agriculture, Forestry and Water Management. 2013. Vol. 10. http://agro.snauka.ru/2013/10/1169. 